\title{
Distinct physical and structural properties of the ovine uterine serpin
}

\author{
Morgan R. Peltier ${ }^{a}$, Tracy R. Grant ${ }^{b}$, Peter J. Hansen ${ }^{\mathrm{a}, *}$ \\ a Department of Animal Sciences, University of Florida, Gainesville, FL 32610-0920, USA \\ b Department of Biochemistry, University of Georgia, Athens, GA 30602, USA
}

Received 14 December 1999; received in revised form 9 March 2000; accepted 14 March 2000

\begin{abstract}
Experiments were performed to examine the relationship between the structure and function of ovine uterine serpin (OvUS). Limited proteolytic digestion of OvUS caused cleavage of the 55-57 kDa OvUS to a $42 \mathrm{kDa}$ product nearly identical in molecular weight to a naturally-occurring breakdown product of OvUS. N-terminal amino acid sequencing and MALDIMS revealed that, unlike other serpins, OvUS was preferentially cleaved at about 70 amino acids upstream of the putative reactive center loop. Analysis of the partially-digested protein by gel filtration chromatography suggested that the C-terminal fragment of the protein was still associated under nondenaturing conditions. Partial digestion of OvUS had no effect on the protein's secondary structure, thermal stability, ability to bind lymphocytes or pepsin, or inhibitory activity towards pepsin or mitogen-induced lymphocyte proliferation. In contrast, mild denaturation of OvUS with $0.5 \mathrm{M}$ guanidine $\mathrm{HCl}$ increased thermal stability. Unlike for other serpins, the increase in thermal stability was lost upon removal of the denaturant. Incubation of OvUS with 100 fold molar excess of a peptide corresponding to the putative $\mathrm{P}_{14}-\mathrm{P}_{2}$ region of the RCL for $24 \mathrm{~h}$ at $37^{\circ} \mathrm{C}$ to induce binary complex formation had no effect on its secondary structure and did not alter the biological activity of the protein. Synthetic peptides corresponding to the putative $\mathrm{P}_{14}-\mathrm{P}_{2}$ region and the $\mathrm{P}_{7}-\mathrm{P}_{15^{\prime}}$ region of the RCL were not inhibitory to pepsin activity or lymphocyte proliferation. Taken together, these results indicate that the conformation of OvUS is distinct from the prototypical serpin because conditions that lead to the large-scale conformational change in other serpins such as antithrombin III and $\alpha_{1}$-antitrypsin do not cause similar changes in OvUS. Moreover, the putative RCL does not seem to contain the activity required to inhibit lymphocyte proliferation or pepsin activity. (C) 2000 Elsevier Science B.V. All rights reserved.
\end{abstract}

Keywords: Serpin; Uterus; Sheep; Cattle; Immunosuppression; Lymphocyte

\footnotetext{
Abbreviations: OvUS, ovine uterine serpin; DPBS, Dulbecco's phosphate buffered saline; PHA, phytohemagglutinin; RCL, reactive center loop; OVA, ovalbumin; BSA, bovine serum albumin; HEL, hen egg lysozyme; AEBSF, 4-(2-aminoethyl)-benzenesulfonyl-fluoride; BCA, bicinchonic acid; TCA, trichloroacetic acid

* Corresponding author. P.O. Box 110920, University of Florida, Gainesville, FL 32511-0920, USA. Fax: +1-352-392-5595;

E-mail: hansen@animal.ufl.edu
}

\section{Introduction}

The uterus of several ungulate species including the sheep [1], cow [2], and pig [3] secretes a novel member of the serpin superfamily of proteinase inhibitors. The best characterized protein of this family of uterine serpins is the ovine uterine serpin, also called uterine milk protein. Like the other members of the family, secretion of ovine uterine serpin (OvUS) by the uterine endometrium is under the control of progesterone [4-6]. Indeed, OvUS can be 
produced in gram quantities during pregnancy [4] and is the most abundant steroid-induced uterine protein described. The OvUS is a glycoprotein of basic $\mathrm{p} I$ (9.2) that exists in the uterine fluid as a 55 and $57 \mathrm{kDa}$ species as well as in various lower molecular weight forms, especially at $45 \mathrm{kDa}$, that appear to be breakdown products of OvUS [4,7].

The OvUS is similar in amino acid sequence to baboon $\alpha_{1}$-antitrypsin and chicken ovalbumin (OVA) [1]. However, OvUS may function as an inhibitor to aspartic proteinases rather than serine proteinases because inhibitory activity of OvUS was detected against pepsin $\mathrm{A}$ and pepsin $\mathrm{C}$ [2] but not against trypsin, chymotrypsin or elastase [1]. The inhibition of aspartic proteinases by the uterine serpins is consistent with the presence of a conserved KVP in the putative reactive center loop (RCL) which is also present in the propeptide region of the pepsinogens. Another conserved motif in the reactive center loop, VVVK, is structurally similar to pepstatin. The OvUS can also bind to a polymorphic family of inactive aspartic proteinases that are produced by binucleated trophoblast cells of the fetus [2]. One major function of OvUS during pregnancy is to mediate progesterone-induced immunosuppression within the uterus and contribute to tolerance of the fetal allograft. The OvUS is inhibitory to lymphocyte proliferation [8-10], natural killer activity [11] and T-helper dependent antibody production [10].

Serpins contain a characteristic RCL that projects from the protein and acts as peptide bait for proteinases [12-16]. The presence of this loop makes the serpin thermodynamically unstable; typical serpins precipitate upon heating at $60^{\circ} \mathrm{C}$ for $2 \mathrm{~h}$ [12,17-19]. Proteolytic cleavage of the RCL by target or nontarget proteinases results in an increase in thermal stability of the protein and a loss of enzyme inhibitory activity of the serpin $[12,17]$. This shift in biochemical activity upon limited proteolysis involves the insertion of a peptide fragment of the RCL into the protein to form strand 4A [20-24]. Incubation of antithrombin III and plasminogen activator inhibitor-1 in dilute concentrations of guanidine $\mathrm{HCl}$ causes unfolding to allow for insertion of the RCL into the protein in a manner similar to limited proteolysis and results in increased thermal stability and altered biological activity [25]. Similarly, incubation of $\alpha_{1}$-antitrypsin or antithrombin III in 100 fold ex- cess of synthetic RCL peptides for $24 \mathrm{~h}$ at $37^{\circ} \mathrm{C}$ also results in an increase in thermal stability [21,24].

The role of the RCL in biological activity has largely been examined in serpins such as $\alpha_{1}$-antitrypsin and antithrombin III that inhibit proteinases. Much less is known about serpins with other biological functions. The noninhibitory serpin corticosteroid binding globulin seems to function using a similar mechanism of action as the prototypical serpin, $\alpha_{1}$-antitrypsin. Limited proteolysis of corticosteroid binding globulin with neutrophil elastase resulted in an increase in thermal stability and a partial loss of affinity of the protein for cortisol [17]. However, another hormone binding serpin, thyroxine binding globulin, became more thermal stable upon partial digestion with human neutrophil elastase but retained its ability to bind to thyroxine [17]. Unlike $\alpha_{1}$-antitrypsin, angiotensinogen and OVA do not undergo a shift in thermal stability upon limited proteolysis of the RCL [26], even though their tertiary structure is similar to the prototypical serpin.

The sequence of the putative RCL of OvUS and similar proteins found in cattle and pigs is very different from other serpins [1] and no structure-function studies have been performed to evaluate the role of the RCL in biological activity of this unique subclass of serpins. Therefore, several experiments were conducted to determine whether limited proteolysis, mild denaturation with guanidine $\mathrm{HCl}$ or pre-incubation with a peptide corresponding to residues $\mathrm{P}_{14}$ $\mathrm{P}_{2}$ of the RCL would result in a large-scale conformational change in OvUS that has been described for other serpins to produce an inactive protein with altered thermal and optical properties. Additional studies were also performed to determine if synthetic peptides corresponding to the RCL could mimic the biological activity of OvUS with regard to inhibition of pepsin activity and lymphocyte proliferation.

\section{Materials and methods}

\subsection{Reagents}

Dulbecco's phosphate buffered saline (DPBS), tis-

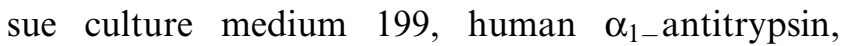
chicken egg OVA, hen egg lysozyme, phytohemag- 
glutinin from Phaseolus vulgaris, penicillin-streptomycin, glutamine, porcine pepsin A, $N, N$-dimethylhemoglobin, pepstatin, heparin, carboxymethyl-Sepharose CL-6B, streptavidin-R-phycoerythrin, pepsin A coupled to agarose, heparin-Sepharose CL-6B and leupeptin were purchased from Sigma (St. Louis, MO). Biotin hydrazine was purchased from Pierce Chemical Company and $\left[\right.$ methyl $\left.-{ }^{3} \mathrm{H}\right]$ thymidine was purchased from ICN (Costa Mesa, CA). The Bradford protein reagent, ampholytes for IEF and silver staining reagents were purchased from Bio-Rad (Hercules, CA). Guanidine $\mathrm{HCl}$ and all other laboratory reagents were purchased from Fisher Scientific (Pittsburg, PA). Synthetic peptides were produced by the Interdisciplinary Center for Biotechnology Research Protein Chemistry Core Laboratory at the University of Florida (Gainesville, FL).

\subsection{Purification of $O v U S$}

The OvUS was purified from uterine fluid collected from unilaterally-pregnant ewes. Prior to breeding, one uterine horn was surgically ligated and the ipsilateral ovary removed as previously described [27] so that the subsequent pregnancy would be restricted to one uterine horn. At approximately day 140 of pregnancy, ewes were slaughtered and fluid was collected from the nongravid uterine horn and stored at $-20^{\circ} \mathrm{C}$ until purification of OvUS by a combination of cation-exchange and gel-filtration chromatography as previously described [4]. After purification, OvUS was buffer-exchanged into DPBS and concentrated using Centricon ultrafiltration devices (Amicon, Beverly, MA). Protein purity was assessed by SDS-PAGE under reducing conditions on $10 \%$ (w/v) polyacrylamide gels and staining with Coomassie Blue R-125.

\subsection{Partial digestion of OvUS with trypsin}

Limited proteolysis of OvUS (3-6 mg ml ${ }^{-1}$ ) was accomplished by incubation of trypsin with OvUS at a 1:60 (w:w) enzyme to serpin ratio in DPBS at $37^{\circ} \mathrm{C}$ for 3.5-4 min. After incubation, the reaction was stopped by adding 10 fold mass excess of 4-(2-aminoethyl)-benzenesulfonyl-fluoride (AEBSF), leupeptin or human $\alpha_{1}$-antitrypsin. Success of the digestion was evaluated by SDS-PAGE using 10 or $12.5 \%$ polyacrylamide gels. To predict the site where OvUS was digested by trypsin, proteins were blotted onto Immobilon- ${ }^{\circledR}$ membranes (Millipore, Bedford, MA) and submitted to the Interdisciplinary Center for Biotechnology Research Protein Chemistry Core Laboratory at the University of Florida (Gainesville, FL) for analysis by $\mathrm{N}$-terminal amino acid sequencing and MALDI-MS.

\subsection{Gel-filtration chromatography of trypsin-digested OvUS}

To determine if the C-terminal fragment of OvUS remains associated after trypsin digestion, trypsin-digested OvUS and undigested OvUS were analyzed by gel-filtration chromatography using a Superdex S-200 column $(1.6 \times 62 \mathrm{~cm})$ on the FPLC system (Pharmacia, Piscataway, NJ). Proteins (1.57 $\mathrm{mg}$ in $500 \mu \mathrm{l} 0.01 \mathrm{M}$ Tris- $\mathrm{HCl}, 0.33 \mathrm{M} \mathrm{NaCl}, \mathrm{pH}$ 8.2) were loaded onto the column and eluted with $0.01 \mathrm{M}$ Tris- $\mathrm{HCl}, 0.33 \mathrm{M} \mathrm{NaCl}, \mathrm{pH} 8.2$ at a $0.5 \mathrm{ml}$ $\mathrm{min}^{-1}$ flow rate. Elution of proteins was monitored by measuring the $\mathrm{A}_{280} ; 0.5 \mathrm{ml}$ fractions were collected in the region of the predicted elution volume $(78.18 \mathrm{ml}$ for OvUS, $83.58 \mathrm{ml}$ for trypsin-digested OvUS). Proteins eluting near the observed OvUS peak were analyzed by SDS-PAGE under reducing conditions using 10\% (w/v) polyacrylamide gels and $50 \mu \mathrm{l}$ sample/lane. Proteins were visualized by silver staining.

\subsection{Preincubation of OvUS with RCL peptide}

Attempts to produce a binary complex form of OvUS were made using procedures similar to those for human $\alpha_{1}$-antitrypsin and antithrombin III $[21,24]$. The OvUS $\left(2 \mathrm{mg} \mathrm{ml}^{-1}\right)$ was incubated with a 100 fold molar excess of a synthetic peptide corresponding to the $\mathrm{P}_{14}-\mathrm{P}_{2}$ region of the putative RCL $\left(\mathrm{OvUS}{ }_{352-364}\right)$ for $24 \mathrm{~h}$ at $37^{\circ} \mathrm{C}$ in $0.1 \mathrm{M}$ Tris- $\mathrm{HCl}$, $\mathrm{pH}$ 8.2. After incubation, the excess peptide was removed by ultrafiltration using $10000 M_{\mathrm{r}}$ Centricon ultrafiltration devices.

\subsection{Thermal stability}

The effects of trypsin digestion, pre-incubation with OvUS ${ }_{352-364}$, and incubation in $0.5 \mathrm{M}$ guanidine 
$\mathrm{HCl}$ on protein thermal stability were tested by heating aliquots of the protein $\left(0.25-0.5 \mathrm{mg} \mathrm{ml}^{-1}\right.$ in DPBS) at $40-90^{\circ} \mathrm{C}$ for $2 \mathrm{~h}$. After heating, the precipitated protein was removed by centrifugation and the concentration of the protein in the supernatant was measured.

\subsection{Circular dichroism}

The relative changes in secondary structure to OvUS (312 nM in $0.01 \mathrm{M}$ sodium phosphate, $\mathrm{pH}$ 8.2) in response to trypsin digestion and incubation with the synthetic RCL peptide OvUS $\mathrm{O}_{352-364}$ were determined by far-UV circular dichroism on a Jasco 710 spectropolarimeter (Tokyo, Japan). Spectra were acquired from 185 to $250 \mathrm{~nm}$ at $0.2 \mathrm{~nm} \mathrm{~s}^{-1}$ and averaged over five scans. Spectra were smoothed using the manufacturer's software and the mean molar ellipticity was calculated after subtracting the background spectrum of the buffer. An unfolding experiment was also performed to determine if OvUS has a two phase unfolding pattern as previously described for antithrombin III [28]. In these experiments, OvUS was incubated in varying concentrations of guanidine $\mathrm{HCl}$ (from 0 to $6 \mathrm{M}$ ) for $2 \mathrm{~h}$ at $25^{\circ} \mathrm{C}$ and the change in mean molar ellipticity at $222 \mathrm{~nm}$ was determined after subtracting the background spectrum for each concentration of guanidine $\mathrm{HCl}$.

\subsection{Pepsin affinity chromatography}

Pepsin-agarose beads were placed in a small $2 \times 0.5$ $\mathrm{cm}$ chromatography column and proteins (1-2 mg) were added in $0.01 \mathrm{M}$ Tris- $\mathrm{HCl}, \mathrm{pH} 8.2$ and eluted with $0.01 \mathrm{M}$ Tris- $\mathrm{HCl}, 3 \mathrm{M} \mathrm{MgCl}_{2}, \mathrm{pH}$ 8.2. In some experiments, $10 \mathrm{mM} \mathrm{KPO}, 0.154 \mathrm{NaCl}, \mathrm{pH} 7.4$ or $0.01 \mathrm{M}$ piperazine buffer, $\mathrm{pH} 10.25$ was used as the column buffer to determine whether binding concentration was electrostatic. The protein of $1 \mathrm{ml}$ fractions of eluate was measured using the bicinchonic acid (BCA) assay.

\subsection{Pepsin activity}

Because the pepsin inhibitory activity of OvUS is very unstable $[2,8]$, all of the pepsin inhibitory assays were performed on freshly purified samples of OvUS that were less than $24 \mathrm{~h}$ old. All treatments were performed in duplicate. Pepsin inhibitory activity was determined by incubating $15 \mu \mathrm{g}$ porcine pepsin A with test proteins in $0.9 \% \mathrm{NaCl}$ in a total volume of $31-67 \mu 1$ at $37^{\circ} \mathrm{C}$ for $15 \mathrm{~min}$. Next, $0.25 \mathrm{ml}$ of $5 \mathrm{mg}$ $\mathrm{ml}^{-1} N, N$-dimethylated hemoglobin in $0.1 \mathrm{M}$ acetate, $\mathrm{pH} 4.5$ was added and the samples were incubated at $37^{\circ} \mathrm{C}$ for $45 \mathrm{~min}$. Proteolysis was then halted by the addition of $0.5 \mathrm{ml} \mathrm{5 \%}(\mathrm{w} / \mathrm{v})$ trichloroacetic acid (TCA). Precipitated proteins were removed by centrifugation at $2000 \times g$ for $10 \mathrm{~min}$ and the $\mathrm{A}_{280}$ of the TCA soluble products was determined. This assay was also used to test the pepsin inhibitory activities of OvUS $352-364$ and $\mathrm{OvUS}_{358-380}$, which correspond to the $\mathrm{P}_{14}-\mathrm{P}_{2}$ and $\mathrm{P}_{7}-\mathrm{P}_{15^{\prime}}$ regions of the putative RCL.

\subsection{Lymphocyte binding}

Binding of OvUS to lymphocytes was measured on Ficoll-Paque purified peripheral blood lymphocytes [29] from adult ewes $(n=2)$ that were suspended at $10^{7}$ cells $\mathrm{ml}^{-1}$ in flow cytometry buffer (DPBS containing $0.1 \%(\mathrm{w} / \mathrm{v})$ bovine serum albumin (BSA) and $2 \mathrm{mM}$ EDTA). Cells were then placed in $12 \times 75 \mathrm{~mm}$ tubes $\left(10^{6}\right.$ cells per tube; two tubes/treatment $)$ on ice. Lymphocytes were incubated with $50 \mu \mathrm{g}$ OvUS, trypsin digested OvUS, OVA or an equivalent amount of trypsin and AEBSF or buffer for $30 \mathrm{~min}$ in a total volume of $111 \mu \mathrm{l}$. Cells were then washed three times with flow cytometry buffer and $0.9 \mu \mathrm{g}$ biotinylated OvUS (20 $\mu$ in DPBS) was added. After 30 min on ice, cells were washed three times with flow cytometry buffer, and resuspended in $10 \mu \mathrm{l}$ streptavidin-Rphycoerythrin $\left(0.5 \mathrm{mg} \mathrm{ml}^{-1}\right)$. After $30 \mathrm{~min}$ on ice, cells were washed three times with cytometry buffer, suspended in $1 \mathrm{ml}$ flow cytometry buffer and analyzed on a Becton Dickinson FACScan flow cytometer. For all analyses, the lymphocyte population was gated by forward and side scatter.

\subsection{Lymphocyte proliferation}

The effects of trypsin digestion and potential binary complex formation on the lymphocyte inhibitory activity of OvUS was tested as previously described using phytohemagglutinin (PHA) as the mitogen to induce lymphocyte proliferation [30]. Peripheral blood mononuclear leukocytes were purified using 
Ficoll-Paque and were plated at $10^{5}$ cells/well in $100 \mu \mathrm{l}$ TCM-199 that was supplemented with $2 \mathrm{mM}$ extra glutamine, $200 \mathrm{U} \mathrm{ml}^{-1}$ penicillin, $0.2 \mathrm{mg} \mathrm{ml}^{-1}$ streptomycin and 5\%(v/v) horse serum in 96 well tissue culture plates. Test proteins $\left(0.5 \mathrm{mg} \mathrm{ml}^{-1}\right.$ final concentration) were then added to each well in a volume of $50 \mu \mathrm{l}$ DPBS and PHA was added at $0.4 \mu \mathrm{g} / \mathrm{well}$ in $10 \mu \mathrm{l}$ DPBS. Cells were placed in a humidified incubator and cultured at $37^{\circ} \mathrm{C}$ in a $5 \%$ $\mathrm{CO}_{2}$ atmosphere for $48-72 \mathrm{~h}$ and then pulsed with $\left[{ }^{3} \mathrm{H}\right]$ thymidine $(0.1 \mu \mathrm{Ci} /$ well $)$ in $40 \mu \mathrm{l}$ modified TCM199 for an additional 14-18 h. Cells were then harvested and the incorporation of $\left[{ }^{3} \mathrm{H}\right]$ thymidine was determined by liquid scintillation counting. For each experiment, cultures were repeated two or three times using lymphocytes from a separate ewe for each replicate. All treatments were done in quadruplicate.

For studies evaluating the effect of preincubation of OvUS with OvUS ${ }_{352-364}$, OvUS $\left(2 \mathrm{mg} \mathrm{ml}^{-1}\right)$ was incubated with 100 fold molar excess peptide and dialyzed as described earlier. After dialysis, $50 \mu \mathrm{l}$ (i.e. $167 \mu \mathrm{g}$ OvUS) of the dialyzed samples were added to each well for all treatments.

In other experiments, peptides corresponding to the $\mathrm{P}_{14}-\mathrm{P}_{2}\left(\mathrm{OvUS}_{352-364}\right)$ or $\mathrm{P}_{7}-\mathrm{P}_{15^{\prime}}\left(\mathrm{OvUS} \mathrm{S}_{358-380}\right)$ regions of the putative RCL were tested for lymphocyte inhibitory activity. Lymphocytes were cultured with $\mathrm{OvUS}_{352-364}$ at concentrations of 8.9 and $89 \mu \mathrm{M}$. These concentrations correspond to concentrations one and 10 times the molar equivalent of $0.5 \mathrm{mg}$ $\mathrm{ml}^{-1}$ OvUS (using 56000 as the molecular weight of OvUS). The peptide $\mathrm{OvUS}_{358-380}$ was tested for lymphocyte inhibitory activity at concentrations ranging from 5 to $320 \mu \mathrm{M}$.

\subsection{Protein assay}

Protein concentration was measured by either the Bradford method [31] or by the BCA modification of the Lowry procedure [32] using BSA as a standard.

\subsection{Statistics}

Data for lymphocyte binding assays and lymphocyte proliferation assays were tested using the General Linear Models procedure of the Statistical Analysis System [33]. Lymphocyte donor (ewe) was
A

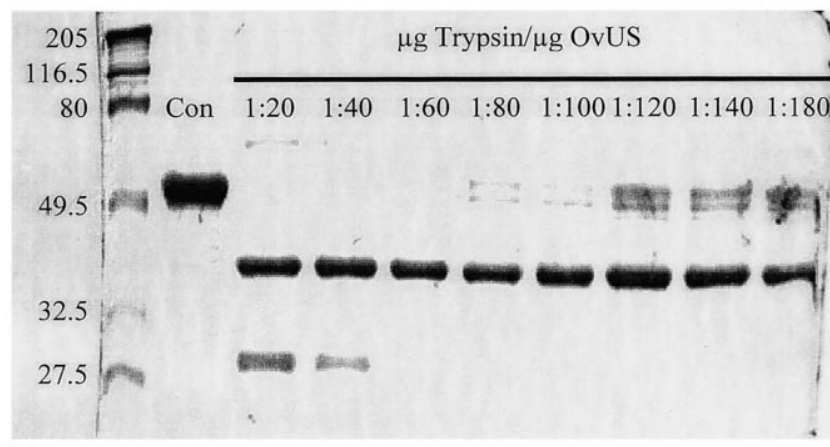

B



Fig. 1. Limited proteolysis of OvUS with trypsin. (A) Effect of trypsin digestion on the molecular weight of OvUS. Trypsin was incubated with OvUS at 1:20-1:180 mass ratios of trypsin: OvUS for $5 \mathrm{~min}$ at $37^{\circ} \mathrm{C}$ in $0.1 \mathrm{M}$ Tris $-\mathrm{HCl}, \mathrm{pH} \mathrm{8.2.} \mathrm{Reaction}$ products were analyzed by SDS-PAGE in the presence of $5 \%$ $(\mathrm{v} / \mathrm{v}) \beta$-mercaptoethanol using a $12.5 \%(\mathrm{w} / \mathrm{v})$ polyacrylamide gel and staining with Coomassie Blue R-250. Molecular weight standards $\left(\times 10^{-3}\right)$ are in the left lane and a control sample of OvUS is in the second lane. (B) Sequencing of OvUS showing the N-terminal amino acid sequence of the naturally occurring $45 \mathrm{kDa}$ OvUS and trypsin-digested OvUS (underlined) and the estimated C-terminal fragment of the protein formed after trypsin digestion (bold type). Change in molecular weight of the serpin upon trypsin digestion was estimated by MALDI-MS and the digestion site was estimated from the published sequence for OvUS (GenBank accession \#J04484). The estimated C-terminal fragment of the protein that is not part of the 42 $\mathrm{kDa}$ product is indicated in bold type. The putative $\mathrm{P}_{1}-\mathrm{P}_{1^{\prime}}$ site is indicated by an asterisk.

considered as a random effect and treatment was considered a fixed effect. Individual means were compared by orthogonal contrasts or the Student-Neuman-Kuels test. All data are reported as leastsquares means \pm S.E.M. 

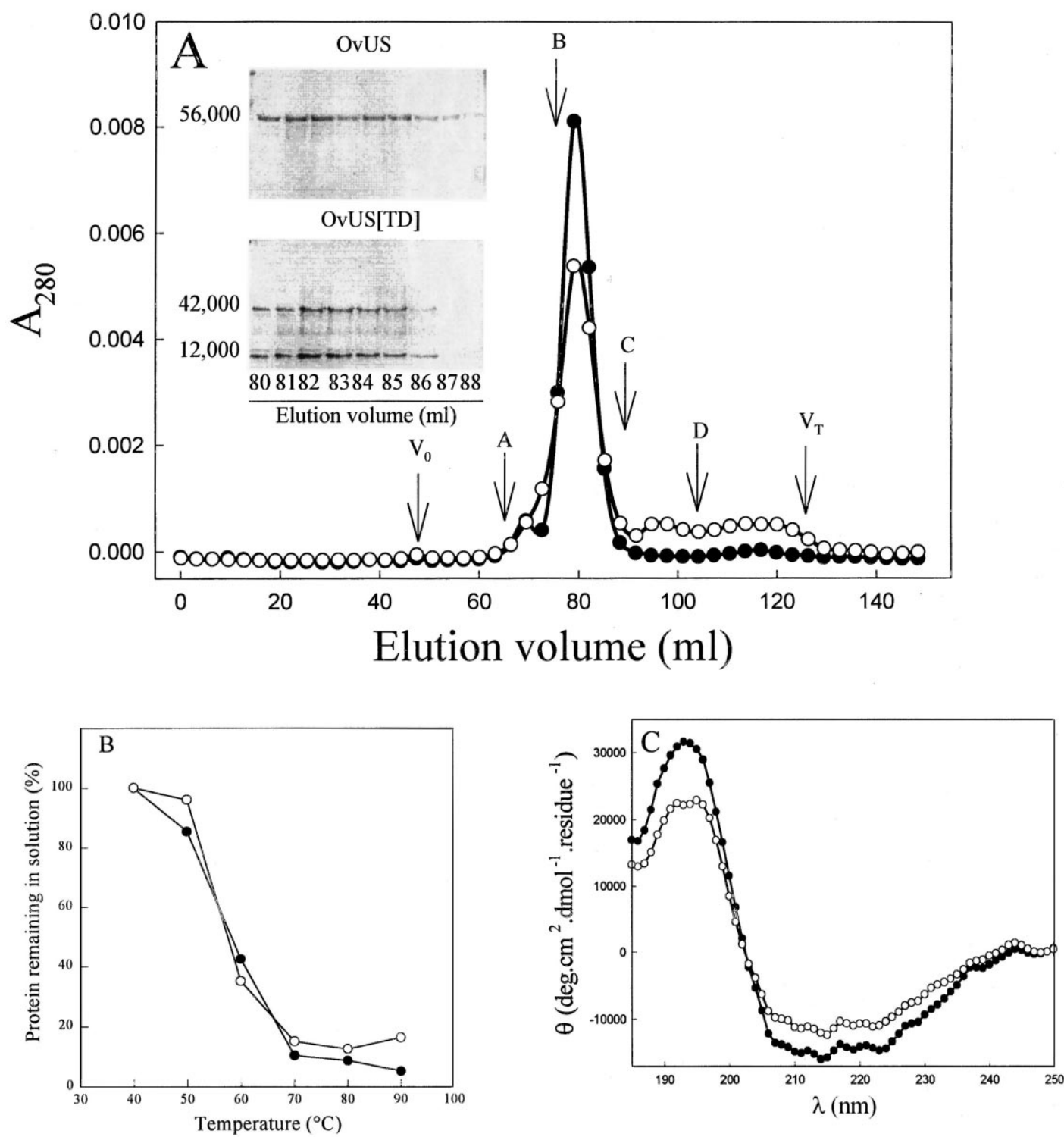

Fig. 2. Effect of limited proteolysis on physical properties of OvUS. (A) Gel filtration chromatography of undigested (•) and trypsin digested $(O)$ OvUS using Superdex S-200HR. The labeled arrows indicate the elution peaks for the void volume $\left(V_{\mathrm{o}}\right)$, BSA $(\mathrm{A})$, hemoglobin (B), OVA (C), HEL (D) and the total volume of the column $\left(V_{\mathrm{T}}\right)$. Inset: Analysis of peak fractions for undigested (OvUS, top gel) and trypsin-digested (OvUS[TD], bottom gel) OvUS. Molecular weights of the resolved proteins are indicated to the left of the gels. (B) Effect of trypsin digestion on the thermal stability of OvUS. Shown is the percentage of undigested ( $(\bullet)$ and trypsin-digested (O) OvUS remaining in the supernatant after heating at various temperatures for $2 \mathrm{~h}$. (C) Effect of limited proteolysis with trypsin on the far UV circular dichroism spectrum of OvUS. Shown is the average circular dichroism spectrum of three batches of protein for undigested $(\bullet)$ and trypsin-digested $(\bigcirc)$ OvUS.

\section{Results}

\subsection{Limited proteolysis experiments}

OvUS exists as a pair of polypeptides of 55 and 57 $\mathrm{kDa}$. Incubation of OvUS with trypsin or pancreatic elastase resulted in a shift in molecular weight from $55-57 \mathrm{kDa}$ to $42 \mathrm{kDa}$ (Fig. 1A). The shift in molecular weight was similar between freshly purified OvUS and stored protein (data not shown). In no case, even with very limited proteolysis, was a digested OvUS identified that was intermediate in 

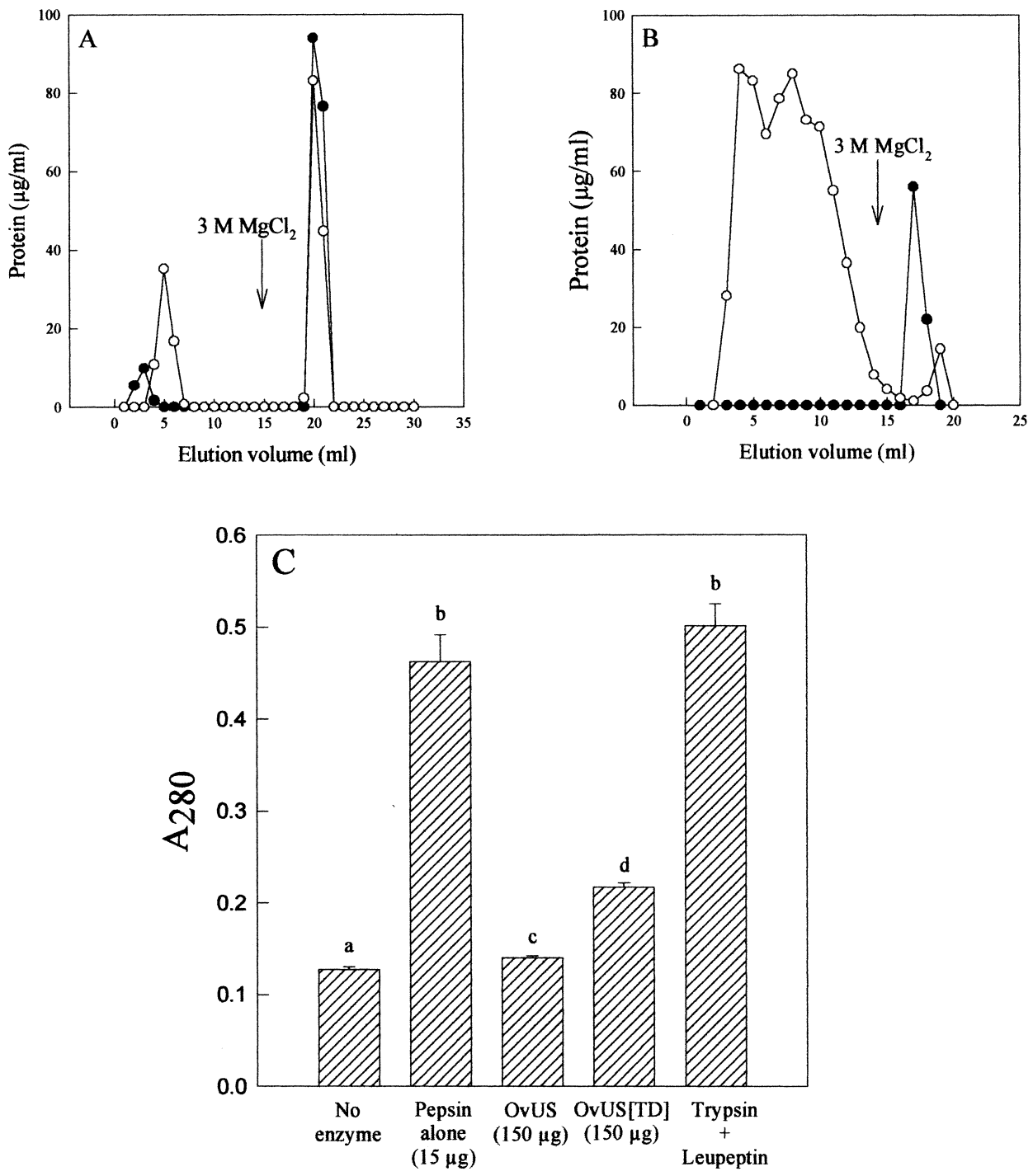

Fig. 3. Effect of limited proteolysis on pepsin binding and inhibitory activity of OvUS. (A) Binding to pepsin. Both a freshly purified sample of OvUS (๑) and trypsin-digested OvUS $(\bigcirc)$ bound to the pepsin-affinity column. (B) Dependence of binding to pepsin upon $\mathrm{pH}$. Note that binding of OvUS occurred at a $\mathrm{pH}$ of $8.0(\bullet)$, where pepsin has a net negative charge and OvUS has a net positive charge, but not at $\mathrm{pH} 10.25(\bigcirc)$ where both proteins are negatively charged. (C) Effect of limited proteolysis on pepsin inhibitory activity of OvUS. Freshly-purified OvUS was digested with trypsin (OvUS[TD]) and tested for pepsin activity against $N, N$-dimethyl-hemoglobin. Undigested OvUS from the same batch of protein (OvUS) and an equivalent amount of trypsin and leupeptin were included as controls. Data are least-squares means \pm S.E.M. of 2-4 replications per treatment. Trypsin digestion had only a slight effect on the anti-pepsin activity of OvUS. Bars having different superscripts are significantly different $(P<0.05)$.

size between the $55-57 \mathrm{kDa}$ protein and the $42 \mathrm{kDa}$ digestion product observed. A similar $45 \mathrm{kDa}$ OvUS is also observed in crude uterine fluid and often copurifies with the $55-57 \mathrm{kDa}$ forms. Both the trypsindigested and the naturally occurring $45 \mathrm{kDa}$ protein had identical $\mathrm{N}$-terminal amino acid sequences that matched the N-terminal sequence of the 55-57 kDa form of OvUS (Fig. 1B). Thus, digestion occurred at the C-terminal end of the protein. More accurate estimates of the change in molecular weight of OvUS caused by trypsin digestion were obtained by MALDI-MS (control $=53196 \mathrm{Da}$; trypsin-digested $=40576 \mathrm{Da})$. The putative location of the cleavage site (between $\mathrm{OvUS}_{295}$ and $\mathrm{OvUS}_{296}$ ) was estimated by matching the change in observed molecular weight with the number of amino acids 
needed to be removed from the C-terminal portion of the protein to obtain this change in size and assuming that the cleavage would occur at the C-terminal side of the nearest arginine or lysine (Fig. 1B). The C-terminal fragment $\left(\mathrm{OvUS} \mathrm{S}_{296-404}\right)$ includes the putative RCL but neither of the putative glycosylation sites.

It is likely that the C-terminal fragment remained associated with the protein because the elution time
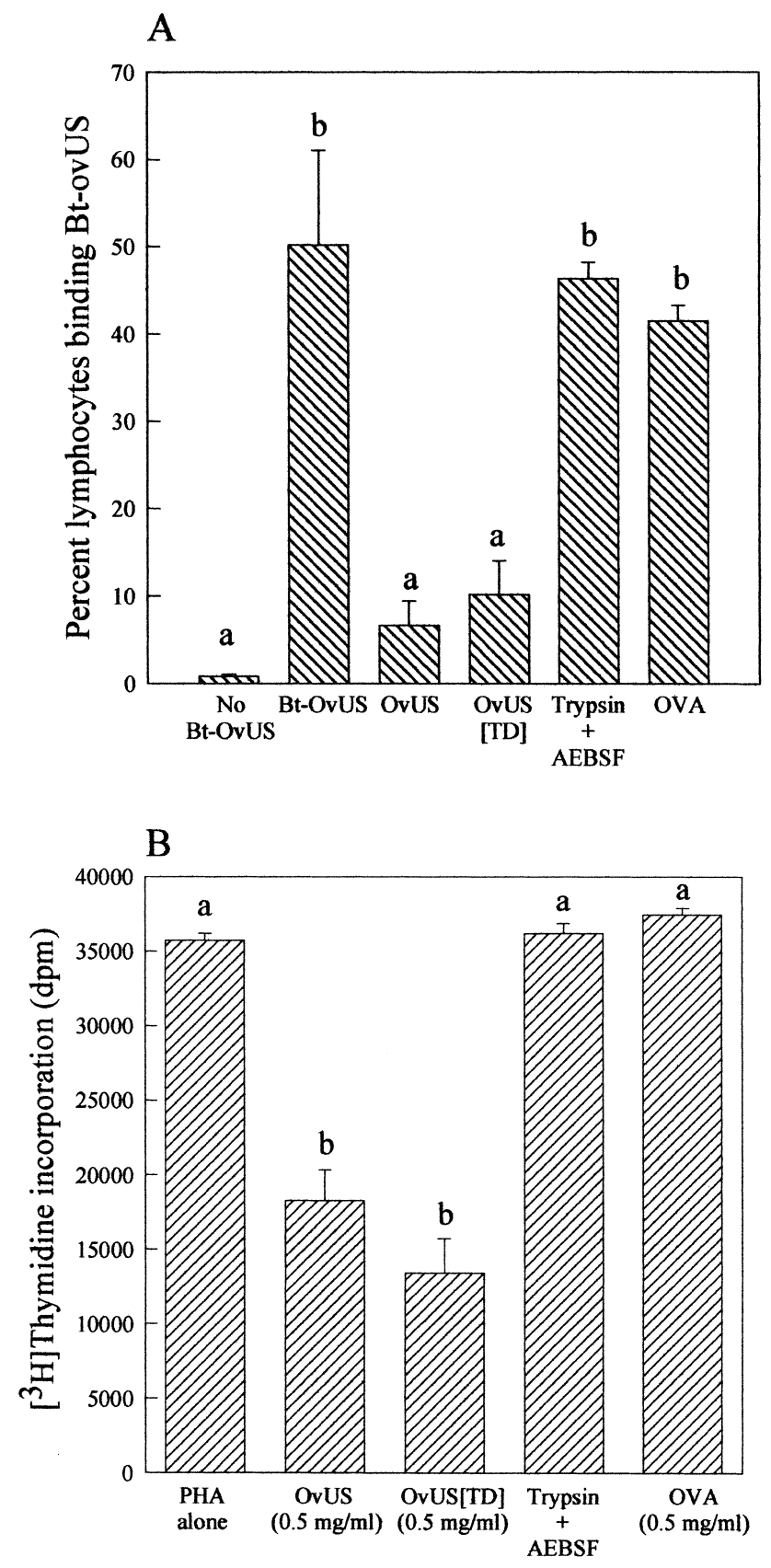

of OvUS on a gel filtration chromatography column was not affected by trypsin digestion (Fig. 2A). On some occasions a $12 \mathrm{kDa}$ band was observed on the gels after trypsin digestion which may correspond to the C-terminal fragment (Fig. 2A). However, not enough of this product could be obtained for analysis by MALDI-MS or N-terminal amino acid sequencing.

The majority of OvUS precipitated when heated at $60^{\circ} \mathrm{C}$ for $2 \mathrm{~h}$ (Fig. 2B). The precipitation of the protein was not due to aggregation via disulfide bond formation because similar results were observed when the thermal stability was determined in the presence of dithiothreitol (data not shown). Limited proteolysis had no effect on the thermal stability of the protein (Fig. 2B). The far UV circular dichroism spectra (Fig. 2C) were similar between undigested OvUS and trypsin-digested OvUS with only a slight decrease in amount of circular dichroism across the spectrum for the trypsin-digested protein.

Binding of OvUS to pepsin [2] has been previously described. Limited proteolysis of OvUS by trypsin digestion had no effect on the binding of OvUS to pepsin (Fig. 3A). Binding of OvUS to pepsin appeared to be electrostatic. Binding occurred in low salt concentrations at $\mathrm{pH}$ 8.0, where pepsin has a net

Fig. 4. Effect of limited proteolysis of OvUS on the binding of OvUS to lymphocytes or inhibition of mitogen-stimulated lymphocyte proliferation. (A) Binding to lymphocytes. The ability of undigested and trypsin-digested OvUS (OvUS[TD]) to compete with biotinylated OvUS (Bt-OvUS) for binding sites on peripheral blood lymphocytes from adult ewes $(n=2)$ was evaluated by flow cytometry. Data are expressed as the percentage of lymphocytes binding biotinylated OvUS. Trypsin digestion did not abrogate the ability of OvUS to compete with biotinylated OvUS for binding to lymphocytes. Neither the control protein, OVA, nor residual trypsin and AEBSF at concentrations used to digest OvUS had any effect on the binding of biotinylated OvUS to lymphocytes. Means with different superscripts are significantly different $(P<0.05)$. (B) Inhibition of lymphocyte proliferation by trypsin-digested OvUS. Trypsin digested OvUS inhibited PHA-stimulated lymphocyte proliferation as effectively as undigested OvUS. Neither trypsin and AEBSF, added at the same concentration present in the trypsin-digested OvUS sample, nor OVA had an effect on mitogenstimulated lymphocyte proliferation. Shown are least-squares means \pm S.E.M. of an experiment using lymphocytes purified from three ewes. Bars with different superscripts are significantly different $(P<0.05)$. 
negative charge and OvUS has a net positive charge, but did not occur at $\mathrm{pH} 10.25$ where both proteins are negatively charged (Fig. 3B). Moreover, binding was lost when buffer with $0.154 \mathrm{M} \mathrm{NaCl}$ was used (data not shown). An alternative explanation is that protein conformation was altered by changes in $\mathrm{pH}$ and salt concentration in a manner that inhibited binding.

Pepsin activity, as measured by the accumulation of TCA soluble $N, N$-dimethyl-hemoglobin digestion products, was inhibited by OvUS. Limited proteolysis by trypsin resulted in only a slight reduction of this activity (Fig. 3C). As a control, trypsin and leupeptin where included as a treatment at the same concentration that was present in the trypsin-digested OvUS sample; neither affected pepsin activity.

Limited proteolysis with trypsin had no effect on the ability for OvUS to compete with biotinylated OvUS (Bt-OvUS) for binding to lymphocytes (Fig. 4A). Both OvUS and trypsin-digested OvUS were equally effective in competing with Bt-OvUS for binding sites. As controls, trypsin and AEBSF at the same concentration that was present in the trypsin-digested OvUS had no effect on the binding of Bt-OvUS to lymphocytes. Similarly, another serpin, OVA, also had no effect on lymphocyte binding to Bt-OvUS, suggesting that the binding sites on lymphocytes are specific for OvUS.

Inhibition of PHA-stimulated $\left[{ }^{3} \mathrm{H}\right]$ thymidine incorporation was also not affected by trypsin digestion (Fig. 4B). Suppression of mitogen-stimulated lymphocyte proliferation was similar for undigested

Fig. 5. Effect of guanidine $\mathrm{HCl}$ on physical properties of OvUS. (A) Effect of guanidine $\mathrm{HCl}$ on thermal stability. Incubation of OvUS in DPBS $+0.5 \mathrm{M}$ guanidine $\mathrm{HCl}(\mathrm{O})$ increased the thermal stability of OvUS relative to protein in DPBS alone (๑). Incubation of OvUS in DPBS $+0.5 \mathrm{M} \mathrm{NaCl}(\mathbf{\square})$ had no effect on thermal stability indicating that the effect of guanidine $\mathrm{HCl}$ was not a nonspecific effect of increased salt concentration. (B) OvUS becomes thermolabile again after removal of guanidine $\mathrm{HCl}$. Shown is the thermal stability of OvUS in DPBS alone $(\bigcirc)$ or DPBS+0.5 M guanidine $\mathrm{HCl}(\bullet)$ followed by desalting on a small $(1 \times 5 \mathrm{~cm}) \mathrm{G}-25$ chromatography column. (C) Unfolding of OvUS with guanidine $\mathrm{HCl}$. There was a single unfolding transition as measured by the loss in CD at $222 \mathrm{~nm} . Y$ is the predicted change in $\mathrm{CD}$ at concentration $X$ of guanidine $\mathrm{HCl}$. and trypsin-digested OvUS. Trypsin and AEBSF at the same concentration used in the trypsin-digested OvUS had no effect on lymphocyte proliferation. Thus the inhibition of lymphocyte proliferation in this treatment was due to cleaved serpin itself. OVA, which had no effect on lymphocyte prolifera-
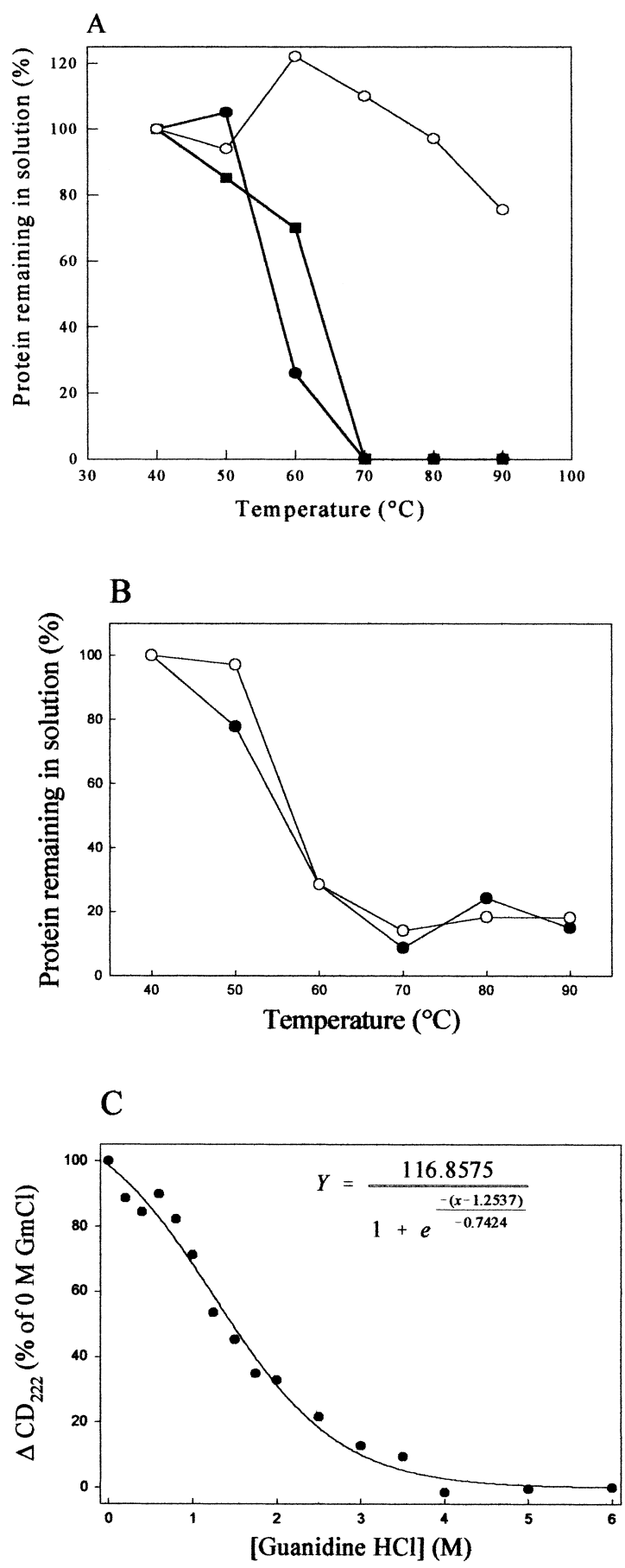
Fig. 6. Effect of pre-incubation of OvUS with a peptide corresponding to residues $\mathrm{P}_{14}-\mathrm{P}_{2}$ of OvUS on physical and lymphocyte proliferation inhibitory activity of OvUS. (A) Thermal stability of control ( () and peptide treated $(\bigcirc)$ samples of OvUS. (B) Far UV CD-spectrum of OvUS. Treatment had little effect on the CD spectrum of OvUS as the spectra were essentially parallel between control $(\bullet)$ and peptide treated $(O)$ proteins. (C) Effect of pre-incubation of OvUS with the synthetic peptide corresponding to $\mathrm{P}_{14}-\mathrm{P}_{2}\left(\mathrm{OvUS}_{352-364}\right)$ on lymphocyte proliferation. This treatment did not block lymphocyte inhibitory activity of OvUS (note both OvUS and peptide+OvUS reduced $\left[{ }^{3} \mathrm{H}\right]$ thymidine incorporation). Neither peptide alone nor the negative controls OVA or HEL reduced proliferation. Shown are least squares means \pm S.E.M. of an experiment utilizing lymphocytes purified from three ewes. Bars with different superscripts are significantly different $(P<0.05)$.

tion, was included as a treatment at the same concentration as OvUS to confirm that the inhibition of lymphocyte proliferation is specific to OvUS.

\subsection{Experiments using mild denaturation with guanidine $\mathrm{HCl}$}

Thermal stability of OvUS was increased in the presence of $0.5 \mathrm{M}$ guanidine $\mathrm{HCl}$ (Fig. 5A). However, removal of guanidine $\mathrm{HCl}$ by desalting with DPBS on a Sephadex G-25 column caused the protein to refold into the thermal-labile state (Fig. 5B). The denaturation curve of OvUS was monophasic with a $15 \%$ decrease in $\alpha$-helical content with 0.4 or $0.8 \mathrm{M}$ guanidine $\mathrm{HCl}$ (Fig. 3D). This large change in conformation with low concentrations of denaturant may explain the shift in thermal stability caused by $0.5 \mathrm{M}$ guanidine $\mathrm{HCl}$.

\subsection{Experiments on OvUS preincubated with a peptide corresponding to the $R C L$}

Pre-incubation of OvUS for $24 \mathrm{~h}$ with a 100 fold molar excess of OvUS $352-364$ (i.e., equivalent to $\mathrm{P}_{14-}$ $\mathrm{P}_{2}$ ) had no effect on the thermal stability of the protein (Fig. 6A) and resulted in only a slight, parallel decrease in the far UV circular dichroism spectrum (Fig. 6B) that is not indicative of the large scale conformational shift described for other serpins. This treatment also had no effect on the ability for OvUS to inhibit lymphocyte proliferation (Fig. 6C). Incubation of OvUS in the presence or absence of



B

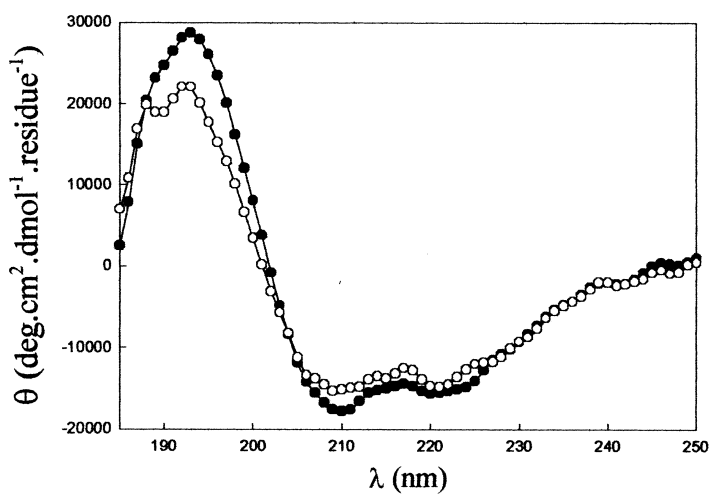

C

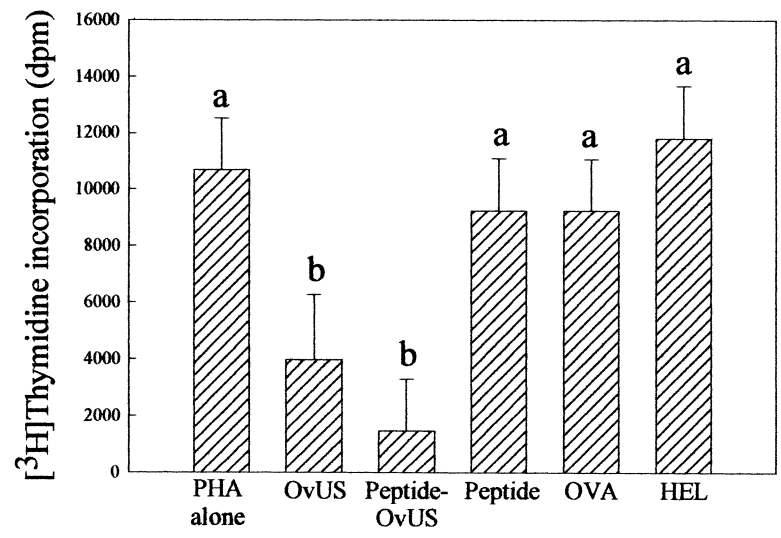

OvUS $_{352-364}$ resulted in a significant suppression of PHA stimulated lymphocyte proliferation when compared to buffer alone, peptide alone, OVA or hen egg lysozyme (HEL). 

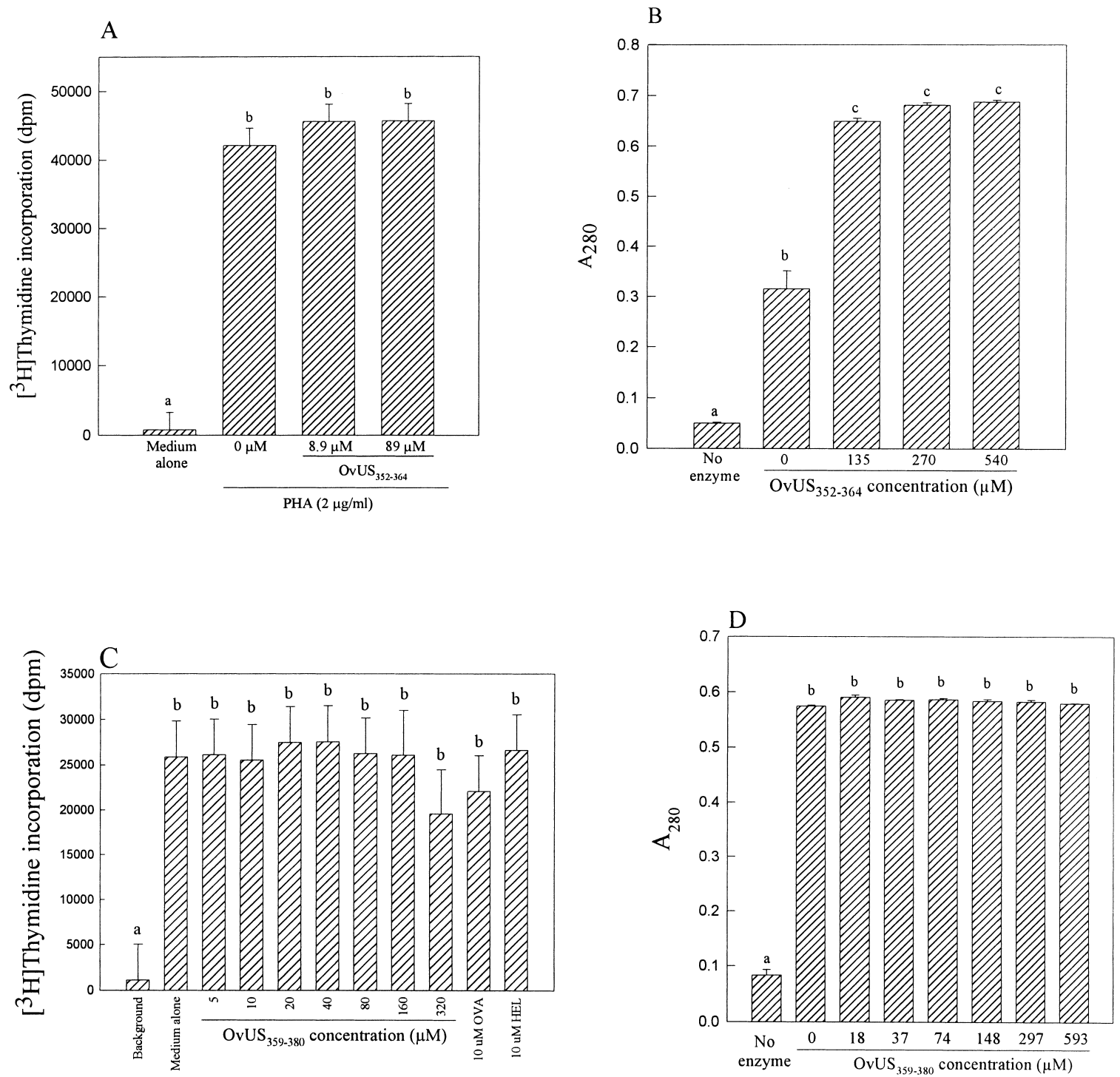

Fig. 7. Biological activity of synthetic peptides corresponding to regions in the putative RCL. A synthetic peptide corresponding to $\mathrm{P}_{14}-\mathrm{P}_{2}\left(\mathrm{OvUS}_{352-364}\right)$ was tested for inhibition of PHA-stimulated lymphocyte proliferation (A) and inhibition of pepsin activity (B). Another peptide, OvUS $359-380$, that corresponds to a larger region of the RCL, $\left(\mathrm{P}_{7}-\mathrm{P}_{15^{\prime}}\right)$ was also tested for inhibition of PHA stimulated lymphocyte proliferation (C) and pepsin activity (D). Neither peptide shared the biological activities of purified OvUS. Negative controls added in (C) are OVA and HEL. Data are least-squares means \pm S.E.M. for $2-4$ observations per treatment. Bars with different superscripts are significantly different $(P<0.05)$.

\subsection{Peptides corresponding to the RCL of OvUS do not inhibit pepsin activity or lymphocyte proliferation}

Neither OvUS ${ }_{352-364}$ nor OvUS $_{359-380}$ (corresponding to the $\mathrm{P}_{14}-\mathrm{P}_{2}$ and $\mathrm{P}_{7}-\mathrm{P}_{15^{\prime}}$ region of the putative RCL, respectively) were inhibitory to lymphocyte proliferation (Fig. 7A,C) or pepsin activity (Fig. $7 \mathrm{~B}, \mathrm{D})$ at molar concentrations equal to or greater than the concentration at which these effects are observed for OvUS. Additionally, pepstatin was not inhibitory to lymphocyte proliferation when tested at equimolar levels to OvUS (data not shown).

\section{Discussion}

The underlying hypothesis for the present studies 
was that, like other serpins, OvUS requires an intact RCL for biological activity. There were two observations supporting this hypothesis. First, a $45 \mathrm{kDa}$ variant of OvUS is present in the uterus during pregnancy $[1,4,7]$ which may represent a partially cleaved form of OvUS. Secondly, OvUS has pepsin-inhibitory activity [2] and it has been hypothesized that this activity is due to the presence of KVP and VVVF residues in the putative RCL [2]. However, several lines of evidence indicate that the conformation of the OvUS is different from prototypical serpins and that the RCL, if existing at all, has different properties than for other serpins. Moreover, studies using peptides corresponding to domains of the putative RCL of OvUS suggest that biological activity of OvUS (i.e., pepsin inhibitory activity and lymphocyte inhibition) does not reside in this region of the protein.

The prototypical serpin inhibits its target proteinase by a characteristic mechanism which results in a partial cleavage at the reactive center, an increase in thermal stability and a loss in biological activity of the serpin [12,17]. Much of the information concerning the role of the RCL in the structure and function of serpins has been determined for only a few serpins that have a target serine proteinase [34,35]. Much less is known about the role of the RCL in serpins with other biological properties. Some such as corticosteroid binding globulin require an intact RCL for biological activity [17] whereas others such as thyroxine binding globulin [17] and pigment epithelium derived factor [18] do not. The RCL of serpins is exposed and is therefore preferentially cleaved by nontarget as well as target proteinases [24]. For example, the RCL of $\alpha_{1}$-antitrypsin was preferentially degraded by the nontarget proteinases, papaya proteinase IV, papain, thermolysin, and bovine $\alpha$-chymotrypsin [24]; the RCL of antithrombin III was cleaved by human neutrophil elastase and thermolysin [24]; the RCL of thyroxine binding globulin and corticosteroid binding globulin was cleaved by neutrophil elastase [17]; the RCL of OVA was cleaved by pancreatic elastase and subtilisin Carlsberg [26]; the RCL of maspin was cleaved by chymotrypsin, elastase, plasmin, thrombin, trypsin, tissue plasminogen activator, and urokinase-type plasminogen activator [19]; the RCL of pigment epithelium derived factor was cleaved by trypsin and chymotrypsin [18] and the RCL of angiotensinogen was cleaved by neutrophil elastase [26]. Therefore, limited proteolytic digestion of OvUS with trypsin or elastase would be expected to result in the formation of a similarly cleaved serpin.

In contrast to results with many other serpins, OvUS was cleaved by trypsin and elastase at a site about 70 amino acids upstream of the putative RCL and did not cause any changes in the physical or biological properties of the protein. There are two explanations for this observation, both of which lead to the conclusion that OvUS has a conformation that is distinct from the prototypical serpin. One possibility is that the putative RCL for OvUS identified by amino acid alignment with other serpins [1] is incorrect and the RCL is located near where the trypsin and elastase cleaved the OvUS (i.e., at a position about 70 amino acids upstream from where the putative RCL was identified). Alternatively, OvUS is in a conformation such that the RCL is inaccessible to proteolytic cleavage. This could occur if OvUS is folded into a latent conformation such as occurs spontaneously for plasminogen activator inhibitor 1 [36] and can be induced for antithrombin [37] and $\alpha_{1}$-antitrypsin [38]. In the latent conformation, there is partial insertion of the RCL into sheet A [25,39] making it inaccessible to proteolysis [37,38,40,41] and preferential cleavage may occur at other regions of the serpin [37,42]. However, unlike previously described serpins in the latent conformation [25,37, 38,43 ], OvUS was biologically active, thermal labile and easily denatured with low concentrations of chaotrophic agents.

OvUS appears to be similar to other serpins with regard to amino acid sequence [1]; the circular dichroism spectrum was also similar for prototypical serpins such as antithrombin III and $\alpha_{1}$-antitrypsin [44-46] and only slightly different from OVA [47-49]. This suggests that the overall structure of OvUS is not very different from the protypical serpins and that slight differences in amino acid sequence may be responsible for the non-native conformation of OvUS. Further work using X-ray crystallography will be necessary to determine if OvUS contains a RCL that is similar to other serpins, if it is accessible to proteinases and the portion of the amino acid sequence that encodes it. Although cleavage occurs near an insertion sequence (KINLKHLLP) that is 
not present in the bovine or porcine uterine serpins, it is unlikely that the cleavage of OvUS is dependent on this region of the protein because naturally-occurring breakdown products of uterine serpin are also present in bovine uterine fluid [50].

For the prototypical serpin, cleavage at the RCL leads to a large conformational change in the protein caused by insertion of the C-terminal peptide fragment into the protein to form strand 4A [20]. This conformational change is associated with increased thermal stability and a loss of biological activity $[12,17]$. In contrast, there was no effect of limited digestion of OvUS with trypsin on thermal lability, secondary structure as determined by circular dichroism, or on biological activity. The absence of an effect of limited proteolysis of OvUS on the physical and biological properties of the protein likely reflects the fact that the $\mathrm{C}$-terminal peptide released by proteolysis is too large or otherwise unable to interact with the larger fragment of the protein. Limited enzymatic digestion also failed to alter thermal stability of other serpins including OVA [26], maspin [19], angiotensinogen [26] and pigment epithelium-derived factor [18]. Similarly, some non-inhibitory serpins such as corticosteroid binding globulin [17] become inactive following limited proteolysis whereas others such as thyroxine binding globulin [17] and pigment epithelium-derived factor remain active [18].

Because inactivation of serpins involves insertion of the N-terminal region of the cleaved RCL into the A-sheet, it is possible to mimic the effects of limited proteolysis by incubating the serpin with a synthetic peptide at 100 fold molar excess for $24 \mathrm{~h}$ at $37^{\circ} \mathrm{C}$ [24]. Attempts to produce a binary complex (serpin with synthetic RCL peptide inserted) of OvUS were made by incubation of OvUS with a synthetic peptide corresponding to the putative $\mathrm{P}_{14}-\mathrm{P}_{2}$ region of the protein for $24 \mathrm{~h}$ at $37^{\circ} \mathrm{C}$. However, no changes in OvUS thermal stability, circular dichroism spectra or ability to inhibit lymphocyte proliferation were observed. Although the conditions used in this experiment are favorable to binary complex formation in other serpins, it is uncertain whether or not the peptide inserted into the protein (as may not happen if, for example, the identification of the putative $\mathrm{RCL}$ is incorrect) or if the peptide inserted but failed to alter biological and optical properties of OvUS. If OvUS is in a latent conformation, insertion of the peptide would be blocked by the partially inserted RCL.

Even though the C-terminal fragment of OvUS does not appear to undergo insertion into the larger fragment of OvUS, it was observed that the C-terminal fragment remained associated with OvUS after cleavage. The nature of the association of this $\mathrm{C}$ terminal fragment after digestion of the protein is uncertain but may be due to hydrogen bonding or hydrophobic interactions between regions of the protein. However, it is unlikely that the association is due to disulfide linkages because no cystines are present on the predicted C-terminal fragment. It is also possible that the $\mathrm{C}$-terminal fragment does insert but unlike other serpins, no changes in the biochemical properties of the protein are associated with this insertion. Like many other serpins [12,17-19,25, 26,51], OvUS was thermal labile with most of the activity precipitating at $60^{\circ} \mathrm{C}$.

Serpins can also be placed into an inactive, thermal stable conformation by incubation in a dilute solution of guanidine $\mathrm{HCl}$ [25]. Mild denaturation of the protein with $0.5 \mathrm{M}$ guanidine $\mathrm{HCl}$ increased thermal stability of OvUS. This phenomenon may represent unfolding of the $\mathrm{N}$-terminal portion of the protein which would have allowed portions of the RCL to fold into the protein and cause a change in conformation of the remainder of the protein as previously described for other serpins [25]. However, this change in conformation, whether due to unfolding of the RCL or another part of the protein, was unstable and dependent on the presence of guanidine $\mathrm{HCl}$ because removal of guanidine $\mathrm{HCl}$ by desalting resulted in a return to the thermal-labile state. Other serpins such as antithrombin III and $\alpha_{1}$-antitrypsin remained in a thermal-stable state after removal of guanidine $\mathrm{HCl}$ by dialysis [25]. Unlike antithrombin III, which demonstrated a two phase denaturation curve with inflections at 0.7 and $4 \mathrm{M}$ guanidine $\mathrm{HCl}$ [28], OvUS displayed only a single, continuous unfolding pattern that resulted in observable changes in circular dichroism at $222 \mathrm{~nm}$ with only $0.2 \mathrm{M}$ guanidine $\mathrm{HCl}$. This suggests that OvUS is very unstable to guanidine $\mathrm{HCl}$ denaturation and that, unlike antithrombin III, there is no well defined intermediate phase in the unfolding curve.

Previous experiments have demonstrated that OvUS was not inhibitory to common serine protein- 
ases such as trypsin, chymotrypsin and elastase [1]. A more recent report demonstrated that OvUS was inhibitory to pepsin A and pepsin C [2]. Inhibitory activity to these aspartic proteinases was attributed to motifs in the RCL that were similar to pepstatin and the propepsinogens (KVP and VVVK). We tested the hypothesis that peptides corresponding to these regions would mimic OvUS with regard to inhibition of pepsin activity and possibly mitogenstimulated lymphocyte proliferation. However, synthetic peptides made to the $\mathrm{P}_{14}-\mathrm{P}_{2}$ or $\mathrm{P}_{7}-\mathrm{P}_{15^{\prime}}$ regions of the putative RCL had no observable biological activity even at concentrations that were 10 fold or more excess the required molar concentrations for activity of OvUS. These results suggest that the putative RCL of OvUS may not be solely responsible for the biological activity of the protein with regards to inhibition of pepsin activity and mitogen-stimulated lymphocyte proliferation. Alternatively, the sequences within the putative RCL may be responsible for biological activity but the peptides not be properly folded into the proper conformation to inhibit lymphocyte proliferation or pepsin activity.

Based on the above experiments, it is concluded that OvUS has a conformation that is distinct from the prototypical serpin because conditions that induce a large scale conformational shift in other serpins do not cause a similar shift in serpin structure. Thus, the uterine serpins, which recent results indicate diverged from other serpins prior to the divergence of mammals from other vertebrates [52], most likely have developed structural requirements for biological activity that are distinct from those used to inhibit proteinases. This divergence in structure may reflect the unique biological role of the protein during pregnancy. Exactly how the structure of OvUS differs from the other serpins and whether or not the protein is folded into a latent, but thermal-labile and biologically-active conformation requires further experiments using methods such as $\mathrm{X}$-ray crystallography.

\section{Acknowledgements}

This work is No R-06573 of the Florida Agricultural Experiment Station and was funded in part by USDA NRICGP Grant No. 96-35203-3304. The au- thors wish to thank Mr. Alfred C. Chung and Mr. Edy Segura of the ICBR Protein Chemistry Core Laboratory for production of the synthetic peptides, MALDI-MS and N-terminal amino acid sequencing; Dr. Leon Dure of the Biochemistry Department at the University of Georgia for providing the instrumentation for the circular dichroism experiments; Mr. Neal Benson and Mrs. Melissa Chen of the ICBR Flow Cytometry Core Laboratory for assistance with flow cytometry; and Dr. Wen-Jun Liu and Miss Susan Gottshall for assistance with purification of OvUS.

\section{References}

[1] N.H. Ing, R.M. Roberts, J. Biol. Chem. 264 (1989) 33723379.

[2] N. Mathialagan, T.R. Hansen, Proc. Natl. Acad. Sci. USA 93 (1996) 13653-13658.

[3] P.V. Malathy, K. Imakawa, R.C. Simmen, R.M. Roberts, Mol. Endocrinol. 4 (1990) 428-440.

[4] R.J. Moffatt, F.W. Bazer, P.J. Hansen, P.W. Chun, R.M. Roberts, Biol. Reprod. 36 (1987) 419-430.

[5] N.H. Ing, H. Francis, J.J. McDonnell, J.F. Amann, R.M. Roberts, Biol. Reprod. 41 (1989) 643-654.

[6] D.C. Stephenson, M.V. Leslie, B.G. Low, G.R. Newton, P.J. Hansen, F.W. Bazer, Domest. Anim. Endocrinol. 6 (1989) 349-362.

[7] P.J. Hansen, N.H. Ing, R.J. Moffatt, G.A. Baumbach, P.T. Saunders, F.W. Bazer, R.M. Roberts, Biol. Reprod. 36 (1987) 405-418.

[8] E.C. Segerson, R.J. Moffatt, F.W. Bazer, R.M. Roberts, Biol. Reprod. 30 (1984) 1175-1186.

[9] B. Skopets, P.J. Hansen, Biol. Reprod. 49 (1993) 9971007.

[10] B. Skopets, W.J. Liu, P.J. Hansen, Am. J. Reprod. Immunol. 33 (1995) 86-93.

[11] W.J. Liu, P.J. Hansen, Biol. Reprod. 49 (1993) 1008-1014.

[12] R.W. Carrell, M.C. Owen, Nature 317 (1985) 730-732.

[13] J. Travis, Adv. Exp. Med. Biol. 425 (1997) 1-4.

[14] J. Travis, A. Guzdek, J. Potempa, W. Watorek, Biol. Chem. Hoppe Seyler 371 (Suppl) (1990) 3-11.

[15] P. Gettins, P.A. Patston, M. Schapira, Hematol. Oncol. Clin. North Am. 6 (1992) 1393-1408.

[16] P. Gettins, P.A. Patston, M. Schapira, Bioessays 15 (1993) 461-467.

[17] P.A. Pemberton, P.E. Stein, M.B. Pepys, J.M. Potter, R.W. Carrell, Nature 336 (1988) 257-258.

[18] S.P. Becerra, A. Sagasti, P. Spinella, V. Notario, J. Biol. Chem. 270 (1995) 25992-25999.

[19] P.A. Pemberton, D.T. Wong, H.L. Gibson, M.C. Kiefer, P.A. Fitzpatrick, R. Sager, P.J. Barr, J. Biol. Chem. 270 (1995) 15832-15837. 
[20] H. Loebermann, R. Tokuoka, J. Deisenhofer, R. Huber, J. Mol. Biol. 177 (1984) 531-557.

[21] A.J. Schulze, U. Baumann, S. Knof, E. Jaeger, R. Huber, C.B. Laurell, Eur. J. Biochem. 194 (1990) 51-56.

[22] A.J. Schulze, P.W. Frohnert, R.A. Engh, R. Huber, Biochemistry 31 (1992) 7560-7565.

[23] A.J. Schulze, R. Huber, W. Bode, R.A. Engh, FEBS Lett. 344 (1994) 117-124.

[24] W.S. Chang, M.R. Wardell, D.A. Lomas, R.W. Carrell, Biochem. J. 314 (1996) 647-653.

[25] R.W. Carrell, D.L. Evans, P.E. Stein, Nature 353 (1991) 576-578

[26] P.E. Stein, D.A. Tewkesbury, R.W. Carrell, Biochem. J. 262 (1989) 103-107.

[27] F.W. Bazer, R.M. Roberts, S.M. Basha, M.T. Zavy, D. Caton, D.H. Barron, J. Anim. Sci. 49 (1979) 1522-1527.

[28] G.B. Villanueva, N. Allen, J. Biol. Chem. 258 (1983) 1101011013.

[29] B.G. Low, P.J. Hansen, M. Drost, J. Reprod. Immunol. 19 (1991) 25-41.

[30] S.L. Gottshall, P.J. Hansen, Zentralbl. Veterinarmed. B 41 (1994) 541-547.

[31] M.M. Bradford, Anal. Biochem. 72 (1976) 248-254.

[32] P.K. Smith, R.I. Krohn, G.T. Hermanson, A.K. Mallia, F.H. Gartner, M.D. Provenzano, E.K. Fujimoto, N.M. Goeke, B.J. Olson, D.C. Klenk, Anal. Biochem. 150 (1985) $76-85$.

[33] SAS. 5.0 Ed., Statistical Analysis System Institute, Cary, NC, 1990.

[34] R. Huber, R.W. Carrell, Biochemistry 28 (1989) 8951-8966.

[35] J. Potempa, E. Korzus, J. Travis, Biol. Chem. 269 (1994) $15957-15960$.

[36] C.M. Hekman, D.J. Loskutoff, J. Biol. Chem. 260 (1985) 11581-11587.

[37] M.R. Wardell, W.-S. Chang, D. Bruce, R. Skinner, A.M. Lesk, R.W. Carrell, Biochemistry 36 (1997) 13133-13142.
[38] D.A. Lomas, P.R. Elliot, W.-S. Chang, M.R. Wardell, R.W. Carell, J. Biol. Chem. 270 (1995) 5282-5288.

[39] J. Mottonen, A. Strand, J. Symersky, R.M.t Swee, D.E. Danley, K.F. Geoghegan, R.D. Gerard, E.J. Goldsmith, Nature 355 (1992) 270-273.

[40] K. Wu, T. Urano, H. Irata, Y. Takada, M. Fujie, M. Shikimori, K. Hashimoto, A. Takada, Blood 86 (1995) 10561061.

[41] D.A. Lawrence, S.T. Olson, S. Palaniappan, D. Ginsburg, J. Biol. Chem. 269 (1994) 27657-27662.

[42] A. Gils, I. Knockaert, P.J. Declerck, Biochemistry 35 (1996) 7474-7481.

[43] K. Katagiri, K. Okada, H. Hattori, M. Yano, Eur. J. Biochem. 176 (1988) 81-87.

[44] S.M. VanPatten, E. Hanson, R. Bernasconi, K. Zhang, P. Manavalan, E.S. Cole, J.M. McPherson, T. Edmunds, J. Biol. Chem. 274 (1999) 10268-10276.

[45] D.B. Hood, J.A. Huntington, P.G.W. Gettins, Biochemistry 33 (1994) 8538-8547.

[46] A.J. Schulze, R. Huber, E. Degryse, D. Speck, R. Bischoff, Eur. J. Biochem. 202 (1991) 1147-1155.

[47] P. Mellet, B. Michels, J.G. Bieth, J. Biol. Chem. 271 (1996) 30311-30314.

[48] Y. Sugimoto, S. Sanuki, S. Ohsako, Y. Higashimoto, M. Kondo, J. Kurawaski, H.R. Ibrahim, T. Aoki, T. Kusakabi, K. Koga, J. Biol. Chem. 274 (1999) 11030-11037.

[49] J.A. Huntington, B. Fan, K.E. Karlsson, J. Deinum, D.A. Lawrence, P.G.W. Gettins, Biochemistry 36 (1997) 54325440.

[50] M.V. Leslie, P.J. Hansen, G.R. Newton, Domest. Anim. Endocrinol. 7 (1990) 517-526.

[51] S.P. Becerra, Adv. Exp. Med. Biol. 425 (1997) 223-237.

[52] M.R. Peltier, Immunoregulatory activity, biochemistry and phylogeny of the ovine uterine serpin, Ph.D. Dissertation, University of Florida, Gainesville, FL, 1999. 